\title{
Effect of Intermittent Low-Frequency Electrical Stimulation on the Rat Gastrocnemius Muscle
}

\author{
Arata Tsutaki, ${ }^{1}$ Riki Ogasawara, ${ }^{2}$ Koji Kobayashi, ${ }^{1}$ Kihyuk Lee, ${ }^{1}$ \\ Karina Kouzaki, ${ }^{1}$ and Koichi Nakazato ${ }^{1}$ \\ ${ }^{1}$ Graduate School of Health and Sport Science, Nippon Sport Science University, 7-1-1 Fukazawa, Setagaya-ku, \\ Tokyo 158-8508, Japan \\ ${ }^{2}$ Faculty of Sport and Health Science, Ritsumeikan University, Shiga, Japan
}

Correspondence should be addressed to Arata Tsutaki; tsutaki.a@gmail.com

Received 28 March 2013; Revised 1 June 2013; Accepted 12 June 2013

Academic Editor: Ashraf S. Gorgey

Copyright (C) 2013 Arata Tsutaki et al. This is an open access article distributed under the Creative Commons Attribution License, which permits unrestricted use, distribution, and reproduction in any medium, provided the original work is properly cited.

Low-frequency neuromuscular electrical stimulation (NMES) has been used as an endurance exercise model. This study aimed to test whether low-frequency NMES increases the phosphorylation of anabolic signaling molecules and induces skeletal muscle hypertrophy, as seen with high-frequency NMES. Using Sprague-Dawley rats, 1 bout of exercise (with dissection done immediately (Post 0 ) and $3 \mathrm{~h}$ (Post3) after exercise) and another 6 sessions of training were performed. All experimental groups consisted of high- and low-frequency stimulation (HFS: $100 \mathrm{~Hz}$; LFS: $10 \mathrm{~Hz}$ ). Periodic acid-Schiff (PAS) staining was conducted to investigate type II fiber activation, and western blot analysis (WB) was conducted to examine whether NMES leads to anabolic intracellular signaling. At first, we examined the acute effect of exercise. PAS staining revealed that glycogen depletion occurred in both type I and type II fibers. WB results demonstrated that p70S6K phosphorylation was significantly increased by HFS, but there was no significant difference with LFS. In contrast, ERK 1/2 phosphorylation was increased by LFS at Post0. In the 6-session training, the wet weight and myofibrillar protein were significantly increased by both HFS and LFS. In conclusion, LFS has a similar anabolic effect for skeletal muscle hypertrophy as HFS, but the mediating signaling pathway might differ.

\section{Introduction}

Skeletal muscle demonstrates high plasticity, responding to external stimuli with morphological changes. Skeletal muscle hypertrophy is a well-known adaptative response to external stimuli, such as resistance training. For humans, the American College of Sports Medicine recommends resistance training of over $70 \%$ of 1 repetition maximum (RM) to lead skeletal muscle hypertrophy [1]. In muscle contraction leading to hypertrophy, type II fibers respond to external stimuli with a greater amount of hypertrophy than do type I fibers [2-4]. Since this process is governed by the size principle, training to induce skeletal muscle hypertrophy demands high force in order to generate type II fibers in voluntary contraction. Conversely, several recent studies have reported that low-intensity ( 30\% 1RM) training, in conjunction with high volume, also causes protein synthesis and skeletal muscle hypertrophy $[5,6]$. The most convincing hypothesis is that the later phases of exhaustive training regimens also recruit type II fibers, regardless of the training intensity. Taken together, the mobilization of type II fibers can be viewed as being critical for the induction of skeletal muscle hypertrophy.

Muscle hypertrophy is caused by a shift of the protein metabolic balance toward an anabolic state. This mechanism is regulated by intracellular signaling. Recent studies have shown that the mammalian target of the rapamycin complex (mTORC) signaling pathway is a key regulator of the protein synthesis rate and muscle size $[7,8]$. Furthermore, the state of phosphorylation of the $70-\mathrm{kDa}$ ribosomal protein $\mathrm{S} 6$ kinase (p70S6K), one of the downstream targets of mTORC signaling, is frequently used as an indicator of traininginduced hypertrophy [9-11]. The mitogen-activated protein kinase (MAPK) cascade has also been reported to be another anabolic pathway that is activated by mechanical stress, such as muscle contraction modes, and metabolic states [12-14]. 
Specifically, the activation of extracellular-signal regulated kinase (ERK) 1/2, but not p38 MAPK, is crucial for the induction of muscle hypertrophy [13]. Taken together, the mTORC1 and ERK 1/2 signaling molecules can be regarded as indicators of training-induced muscle hypertrophy.

Electrical stimulation of the skeletal muscle, typically termed neuromuscular electrical stimulation (NMES), is commonly used clinically to enhance the rehabilitation of skeletal muscle function. The force frequency relationship of NMES indicates that increases in the stimulation frequency result in increased muscle force production [15]. In animal experimental muscle models for skeletal muscle hypertrophy, NMES is generally performed with intermittent and high frequencies (more than $60 \mathrm{~Hz}$ ) [16-19]. On the other hand, when NMES was applied with a lower electrical frequency $(\sim 20 \mathrm{~Hz})$ and long activation times, it led to higher oxidative properties and a fast-to-slow conversion of the muscle fiber phenotype [20]. Therefore, animal experimental models with NMES were used to mimic resistance training and/or endurance exercise in order to test the effects of changes in frequency and stimulation time on muscle hypertrophy [21]. Because of its lower exerted force, lowfrequency NMES has not been used as a stimulator for muscle hypertrophy. Since NMES randomly activates type I and type II fibers, independent of frequency [22], there might be a possibility that low-frequency NMES will lead to muscle hypertrophy in the same way that high-frequency does.

In the present study, we aimed to investigate whether intermittent activation with high $(100 \mathrm{~Hz})$ or low $(10 \mathrm{~Hz})$ frequencies of NMES could induce skeletal muscle hypertrophy in the rat. For this purpose, we conducted 2 experiments. First, we investigated whether acute bouts of exercise induced by low or high electrical frequencies of NMES could sway skeletal muscles toward anabolic states. We conducted histological and western blot analysis, focusing on the intracellular signaling phosphorylations of p70S6K (Thr389) and ERK 1/2 (Thr202/Tyr204). In a second experiment, we subjected rats to 6 sessions of training with low or high electrical frequencies, to investigate whether chronic NMES with different frequencies induces muscle hypertrophy.

\section{Materials and Methods}

2.1. Animal Care. Thirty-six male Sprague-Dawley rats (9 weeks old) were purchased from CLEA Japan (Tokyo, Japan). All animals were housed individually, in a $12 \mathrm{~h}$ light-dark cycle, with the lights kept on from 6:00 pm to 6:00 am, and they were given food and water ad libitum. All procedures used in this study were approved by the Ethical Committee of the Nippon Sports Science University.

2.2. Experimental Design. Rats were divided evenly into a high-frequency electrical stimulation (HFS; $100 \mathrm{~Hz}$ ) group and a low-frequency electrical stimulation (LFS; $10 \mathrm{~Hz}$ ) group. They were then further randomly assigned to the following groups: (1) dissection immediately after training
(Post0 group), (2) dissection $3 \mathrm{~h}$ after training (Post3 group), and (3) dissection after 6 sessions of training (6-session group). Thus, there were 6 groups defined by the frequency of electrical stimulation and the time points at which data were collected ( $n=6$ each group).

2.3. Resistance Training Protocol. Under anesthesia, the right lower leg of each rat was shaved. The rats were then subjected to isometric training by electrical stimulation. Each rat was laid prone on a platform and its right knee was extended with a dynamometer, with the ankle joint positioned at an angle of $90^{\circ}$. The triceps of the right leg muscle was then stimulated (voltage: $30-35 \mathrm{~V}$; pulse duration $4 \mathrm{~ms}$ : frequency: $100 \mathrm{~Hz}$ for HFS group and $10 \mathrm{~Hz}$ for LFS group) with a surface electrode $(7.5 \mathrm{~mm} \times 7.5 \mathrm{~mm})$ that was connected to an electrical stimulator and isolator (Nihon Koden, Japan). The left medial gastrocnemius muscle served as the untrained (UT) control.

For all training sessions, the triceps surae muscle was trained by stimulation for five $3 \mathrm{~s}$ contractions, with a $5 \mathrm{~s}$ interval between each contraction. Four sets in total were performed, with 3 min intervals between each set. All training sessions were conducted on every other day. The training and exercise schedule details are presented in Figure 1.

Animals were sacrificed immediately or at 3 or $24 \mathrm{~h}(6$ sessions) after training. The medial gastrocnemius muscle was removed, weighed, and rapidly frozen in liquid nitrogen, and the right and left medial gastrocnemius muscles were triturated in liquid nitrogen and stored at $-80^{\circ} \mathrm{C}$ until use.

2.4. Periodic Acid-Schiff (PAS) Staining and Immunohistochemistry. To investigate the intramuscular glycogen, we used a commercially available PAS staining kit (Muto Chemical Co., Ltd., Japan) and performed the protocol according to the manufacturer's instruction manual.

To classify the fiber type, $10 \mu \mathrm{m}$ thick muscle cryosections were fixed with $2 \%$ paraformaldehyde and $0.25 \%$ picric acid in $0.1 \mathrm{M}$ phosphate-buffered saline (PBS), for $15 \mathrm{~min}$ at room temperature. Following fixation, the sections were washed in $0.1 \mathrm{M}$ PBS for $15 \mathrm{~min}$ and then postfixed in ice-cold methanol $\left(-20^{\circ} \mathrm{C}\right)$ for $10 \mathrm{~min}$. The sections were then washed 3 times in $0.1 \mathrm{M}$ PBS for $5 \mathrm{~min}$ and blocked in a $0.1 \mathrm{M}$ PBS solution containing 5\% goat serum and $1 \%$ Triton-X-100, for $1 \mathrm{~h}$ at room temperature. After blocking, the primary antibodies were applied (laminin and fast myosin heavy chain; Sigma, USA) over night at $4^{\circ} \mathrm{C}$. On the following day, the sections were washed in $0.1 \mathrm{M}$ PBS, and the secondary antibody was applied overnight at $4^{\circ} \mathrm{C}$. The sections were then washed twice in $0.1 \mathrm{M}$ PBS for $10 \mathrm{~min}$ and subsequently dried. Slides were viewed under a light microscope and muscle fibers were classified as either slow myosin heavy chain (MHC I; black) or fast myosin heavy chain (MHC II; green).

2.5. Western Blotting Analysis. Frozen muscle powder was homogenized in a buffer containing $50 \mathrm{mM}$ Tris- $\mathrm{HCl}(\mathrm{pH}$ 7.5), $1 \mathrm{mM}$ ethylenediaminetetraacetic acid (EDTA), $1 \mathrm{mM}$ ethylene glycol tetraacetic acid, $1 \%$ Triton X-100, 10\% glycerol, protease inhibitor (Roche Applied Science), and phosphatase 


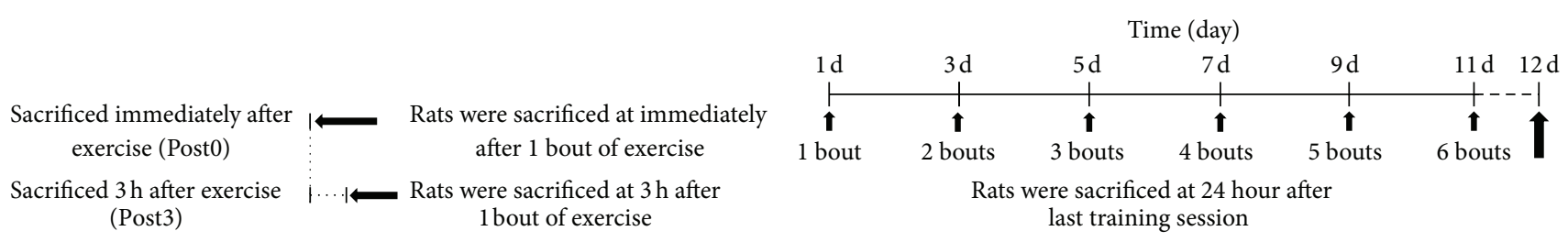

(a)

(b)

FIGURE 1: Training protocol. Schematic diagram representing the details of the experimental schedule in this study. Thirty-six Sprague-Dawley rats were used. The rats were divided into 2 groups: high-frequency electrical stimulation (HFS) and low-frequency electrical stimulation (LFS). Rats were subjected to training on every other day. (a) The left illustration represents 1 bout of exercise. (b) The right illustration represents 6 sessions of a training protocol. The rats in all of the experimental groups $(n=6)$ had their medial gastrocnemius muscle extracted after death.

inhibitor (Thermo Scientific), with sonication for $10 \mathrm{~s}$. Following this, the homogenate was centrifuged for $10 \mathrm{~min}$ at $14,000 \times \mathrm{g}$, at $4^{\circ} \mathrm{C}$, after which the protein extract was mixed with Laemmli sample buffer and then heated to $85^{\circ} \mathrm{C}$. Samples were stored at $-80^{\circ} \mathrm{C}$ until use. Equal amounts were loaded onto a gel for electrophoresis, after which the separated proteins were electroblotted onto a nitrocellulose membrane (GE Healthcare) for $90 \mathrm{~min}$.

After blotting, the membrane was washed in Trisbuffered saline containing $25 \mathrm{mM}$ Tris- $\mathrm{HCl}$ ( $\mathrm{pH}$ 7.4), $150 \mathrm{mM}$ $\mathrm{NaCl}$, and $0.1 \%$ Tween 20 (TBS-T), blocked with $1 \%$ skimmed milk (BD Pharmigen), and then incubated with the desired with the primary antibody overnight at $4^{\circ} \mathrm{C}$. On the next day, the membrane was washed with TBS-T, and peroxidase-conjugated secondary antibodies (Thermo Scientific) were applied for $60 \mathrm{~min}$ at room temperature. Chemiluminescent reagents (Thermo Scientific) were used for the signal detection. The primary antibodies used in this study were phospho-p70S6K (Thr389), phospho-ERK1/2 (Thr202/Tyr204), and $\alpha$-tubulin (all from Cell Signaling Technology, Japan). Images were then captured, and the signals were quantified using the Ez-capture chemiluminescence detector (ATTO) and CS analyzer software (ATTO). All target proteins were normalized $\alpha$-tubulin expression levels.

2.6. Myofibrillar Protein Extraction. Procedures to determine the myofibrillar protein concentration were carried out as described by Karagounis et al. [23]. Approximately $20 \mathrm{mg}$ of powdered muscle was homogenized in a buffer containing $150 \mathrm{mM} \mathrm{NaCl}, 0.1 \%$ Triton-X 100, 20 mM Tris- $\mathrm{HCl}$ (pH 6.8), $50 \mu \mathrm{M}$ dithiothreitol (DTT), $100 \mathrm{mM}$ EDTA, and protease inhibitor (Roche Applied Science) and then centrifuged at $1,600 \times \mathrm{g}$ for $20 \mathrm{~min}$ to produce a myofibrillar pellet. Following removal of the supernatant, the pellet was washed in a lowsalt buffer containing $100 \mathrm{mM} \mathrm{KCl,} 5 \mathrm{mM}$ Tris- $\mathrm{HCl}$ (pH 7.4), and $1 \mathrm{mM}$ DTT and centrifuged for $5 \mathrm{~min}$ at $13,000 \times \mathrm{g}$; the process was repeated twice. The pellet was then washed 2 times in $70 \%$ ethanol. After removal of the ethanol, the pellet was resuspended in $0.3 \mathrm{M} \mathrm{NaOH}$, and an aliquot was removed to determine the protein content by using the Lowry assay (RC DC Assay; BioRad). Protein concentrations were corrected for their bovine serum albumin content.
2.7. Cross-Sectional Area Measurement. To investigate the cross-sectional area (CSA), we used the immunohistochemical technique described by Bloemberg and Quadrilatero [24]. The primary antibodies used were BA-F8 (MHC I), SC-71 (MHC IIa), BF-35 (MHC IIx), and BF-F3 (MHC IIb), all purchased from the Developmental Studies Hybridoma Bank (University of Iowa). The CSA quantification was performed by a computer application (Winroof; Mitsuya Co., Ltd.), with $n=4$ in each group. The CSA of each MHC isoform was counted to 100 fibers per sample.

\subsection{Electrophoresis for Myosin Heavy Chain Composition.} The electrophoretic protocol for separation of the myosin heavy chain isoforms was essentially the same as that described by Mizunoya et al. [25]. Muscle powder was homogenized in a buffer of $10 \%$ sodium dodecyl sulfate (SDS), $40 \mathrm{mM}$ DTT, $5 \mathrm{mM}$ EDTA, $100 \mathrm{mM}$ Tris- $\mathrm{HCl}$ ( $\mathrm{pH}$ 8.0), and protease inhibitor (Roche Applied Science). The homogenates were centrifuged at $15,490 \times \mathrm{g}$ for $5 \mathrm{~min}$ at $4^{\circ} \mathrm{C}$. The supernatant was extracted, and the protein concentration for each sample was determined by ultraviolet absorption spectrophotometry.

Samples were diluted to final protein concentrations of $10-1,280 \mathrm{ng} / \mu \mathrm{L}$ in a mixed sample buffer containing $100 \mathrm{mM}$ DTT, 4.0\% SDS, 160 mM Tris- $\mathrm{HCl}$ ( $\mathrm{pH} 6.8$ ), 43\% glycerol, $0.2 \%$ bromophenol blue, and $\mathrm{dH}_{2} \mathrm{O}$. After boiling, the protein concentrations were adjusted to $20 \mathrm{ng} / \mu \mathrm{L}$ and frozen at $-80^{\circ} \mathrm{C}$ until use.

The separating gel consisted of $30 \%$ glycerol and $8 \%$ acrylamide, and the stacking gel consisted of $30 \%$ glycerol and $4 \%$ acrylamide. Electrophoresis was performed at $4{ }^{\circ} \mathrm{C}$ with a constant voltage of $140 \mathrm{~V}$ for $22 \mathrm{~h}$ (except for the first $40 \mathrm{~min}$, during which the maximal current was limited to $10 \mathrm{~mA}$ to allow stacking gel penetration). The lower running buffer was mixed gently with a magnetic stirrer throughout the entire electrophoresis. After electrophoresis, the gels were stained with a silver staining kit (Silver Stain KANTO III: Kanto Chemicals) and then dried using a Multi gel Dryer (Cosmo Bio Co., Ltd.). Finally, the bands were quantified by densitometry (Cs Analyzer; ATTO).

2.9. Statistical Analysis. The statistical differences between the trained (T) and untrained (UT) legs were determined by paired $t$-tests. Concurrently, the statistical differences 
between HFS and LFS treatments were determined by unpaired $t$-tests. The time course changes in protein expression were examined by a two-way analysis of variance. A post hoc Bonferroni correction was performed using the $t$-test, and all numbers are expressed as the mean $\pm \mathrm{SD}$. $P<0.05$ was considered to denote acceptable significance.

\section{Results}

\subsection{Experiment I: One Bout of Exercise Induced by NMES with Low and High Electrical Frequencies}

3.1.1. Mechanical Parameter of Force Generation. Figure 2 indicates the recorded parameter of force generation from 1 bout of training in the Post 0 and Post 3 groups. Figure 2(a) represents the typical schema of force generation in 1 bout of training. The top figure represents the HFS group and the bottom figure represents LFS group. Figure 2(b) represents the peak torque $(\mathrm{Po})$, and Figure 2(c) indicates the force integral $(\mathrm{mNm} \cdot \mathrm{S})$. Both the Po and force integral in the LFS group were significantly lower than the values in the HFS group $(P<0.001)$.

3.1.2. PAS Staining and Immunohistochemistry. Figure 3 shows the PAS and immunohistochemical staining results for type II fibers of rat skeletal muscle. Figure 3(a) suggests that type II fibers were dominant in the white portion. On the other hand, Figure 3(b) indicates that both type I and type II fibers coexisted in the red portion.

In Figure 3(a), weak PAS staining was observed in the entire region, suggesting that glycogen depletion occurred under both HFS and LFS. The PAS staining in Figure 3(b) shows weakly stained muscle fibers occurring randomly in type I fibers and type II fibers in both high-frequency- and low-frequency-stimulated muscles, suggesting that electrical stimulation recruited both type I and type II fibers, regardless of the frequency.

3.1.3. Time-Course Changes of Protein Expression. Figure 4 surveys the change in protein expression over different time points. Figure 4(a) shows results of the western blot analysis for the state of p70S6K phosphorylation at Thr 389. At both Post0 and Post3, HFS resulted in higher phosphorylation levels than in the UT $(P<0.01$ at Post $0, P<0.001$ at Post3; Post0 versus Post3 $P<0.05)$. However, LFS caused no significant variation over either time point. Moreover, the phosphorylation of p70S6K in the LFS group at Post0 was significantly lower than that attributed to HFS at Post $0(P<$ 0.05).

Figure 4(b) represents the state of ERK $1 / 2$ phosphorylation at Thr202/Tyr204. Following HFS, the phosphorylation levels at Post0 and Post3 remained unchanged. However, at Post0, only LFS training produced significantly higher levels of phosphorylation $(P<0.05)$.
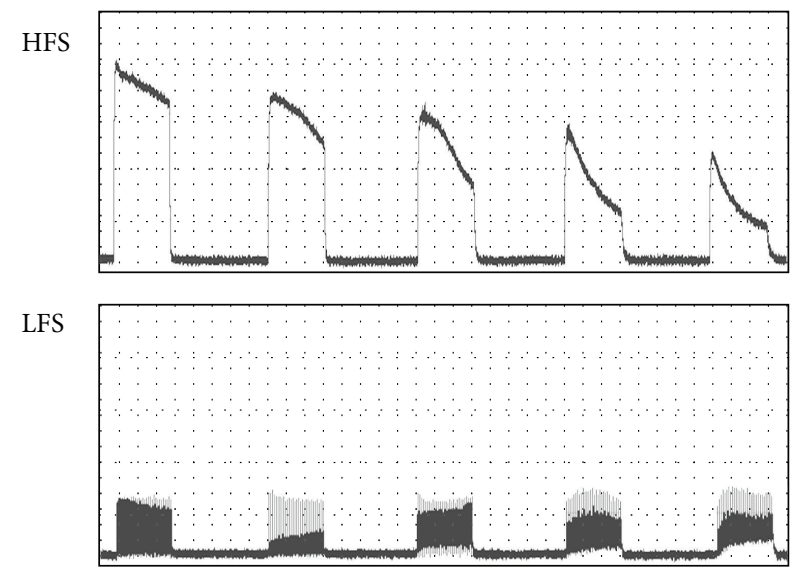

(a)

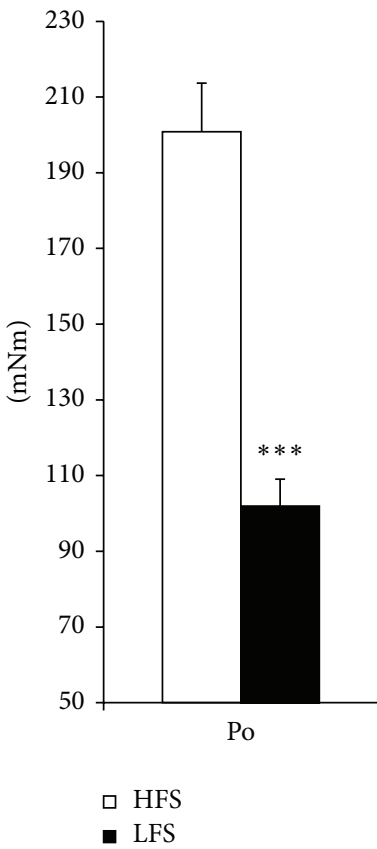

(b)

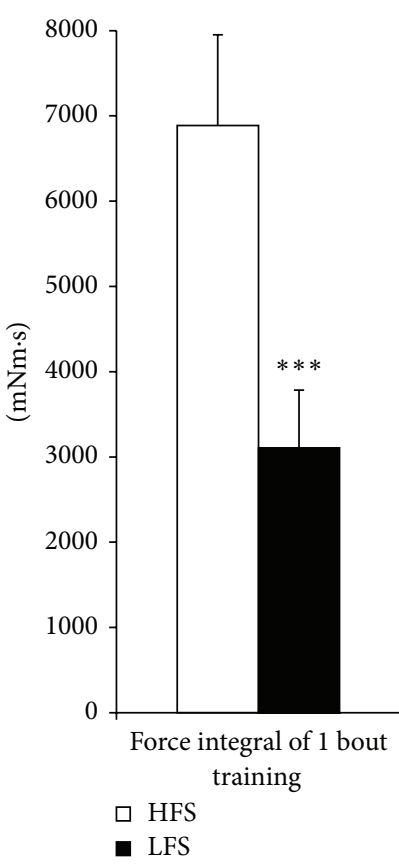

(c)
FIgURE 2: Mechanical parameter of force generation. (a) The typical schema of force generation of each training group. The top figure represents high-frequency electrical stimulation (HFS) and the bottom figure shows low-frequency electrical stimulation (LFS). (b) The peak tetanic torque (Po). (c) The total force generation of 1 bout of exercise. HFS: high-frequency electrical stimulation group. LFS: low-frequency electrical stimulation group. All values are the mean \pm SD. ${ }^{* * *} P<0.001$, versus HFS.

\subsection{Experiment II: Six Sessions of Training with NMES at Differential Frequencies}

3.2.1. Physiological Characteristics after Six Sessions of Training Employing Low and High Electrical Frequencies. Table 1 summarizes the results observed after 6 sessions of training with NMES at differential frequencies. No significant change in body weight occurred between the rats treated with HFS and LFS. However, both the HFS and LFS groups exhibited 

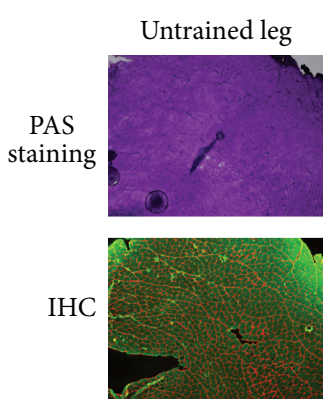

HFS T
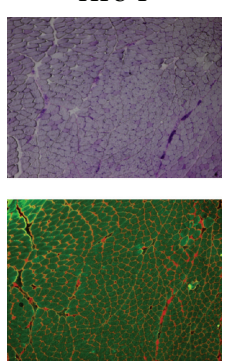

(a)
LFS T
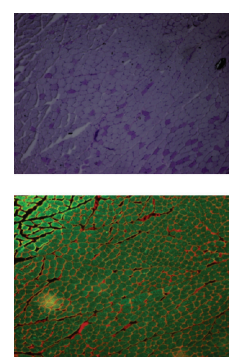
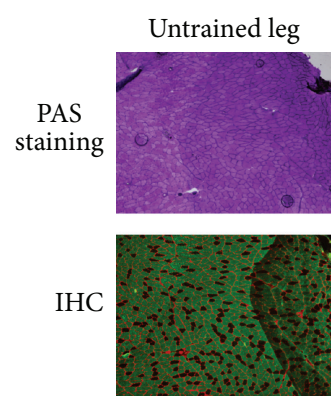

HFS T
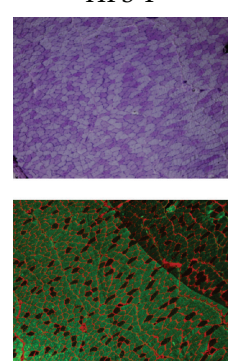

(b)

FIGURE 3: PAS staining and immunohistochemistry. (a) PAS and immunohistochemistry staining patterns in the white portion of the medial gastrocnemius. (b) PAS and immunohistochemistry staining patterns in red portion of the medial gastrocnemius. In PAS staining, the intramuscular glycogen content is indicated by a deep purple color. In immunohistochemistry, myosin heavy chain I (black) and II (green) are indicated. IHC: immunohistochemistry. HFS: high-frequency electrical stimulation group. LFS: low-frequency electrical stimulation group. HFS T and LFS T: the trained leg in HFS and LFS, respectively.

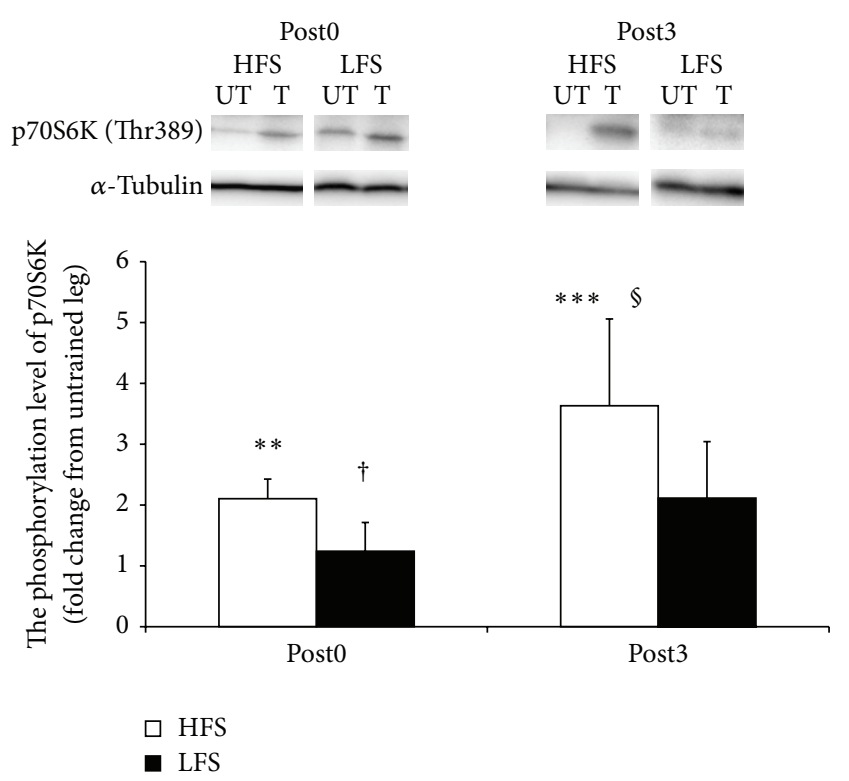

(a)
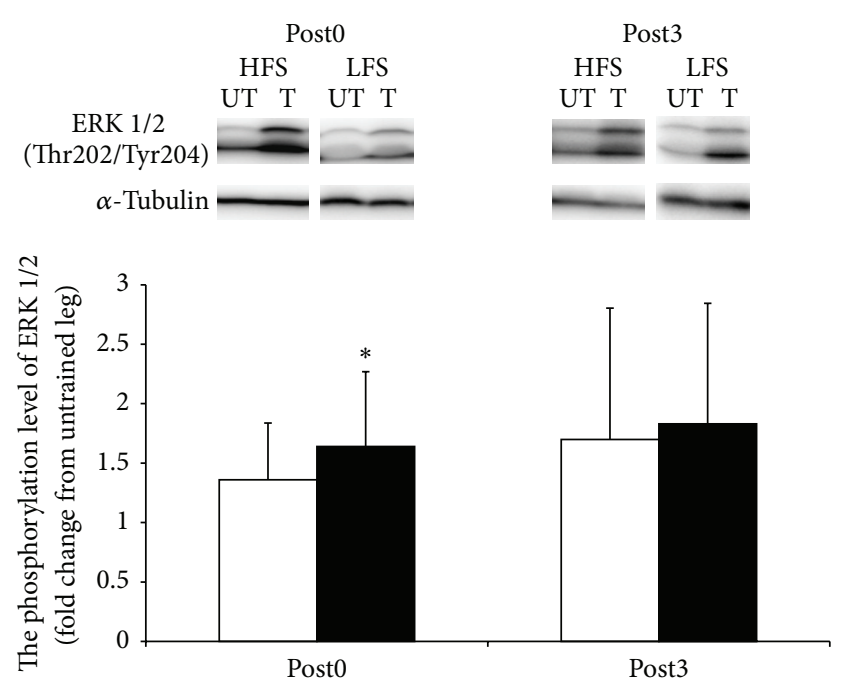

$\square$ HFS

- LFS

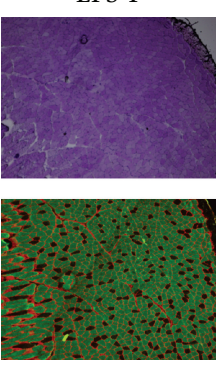



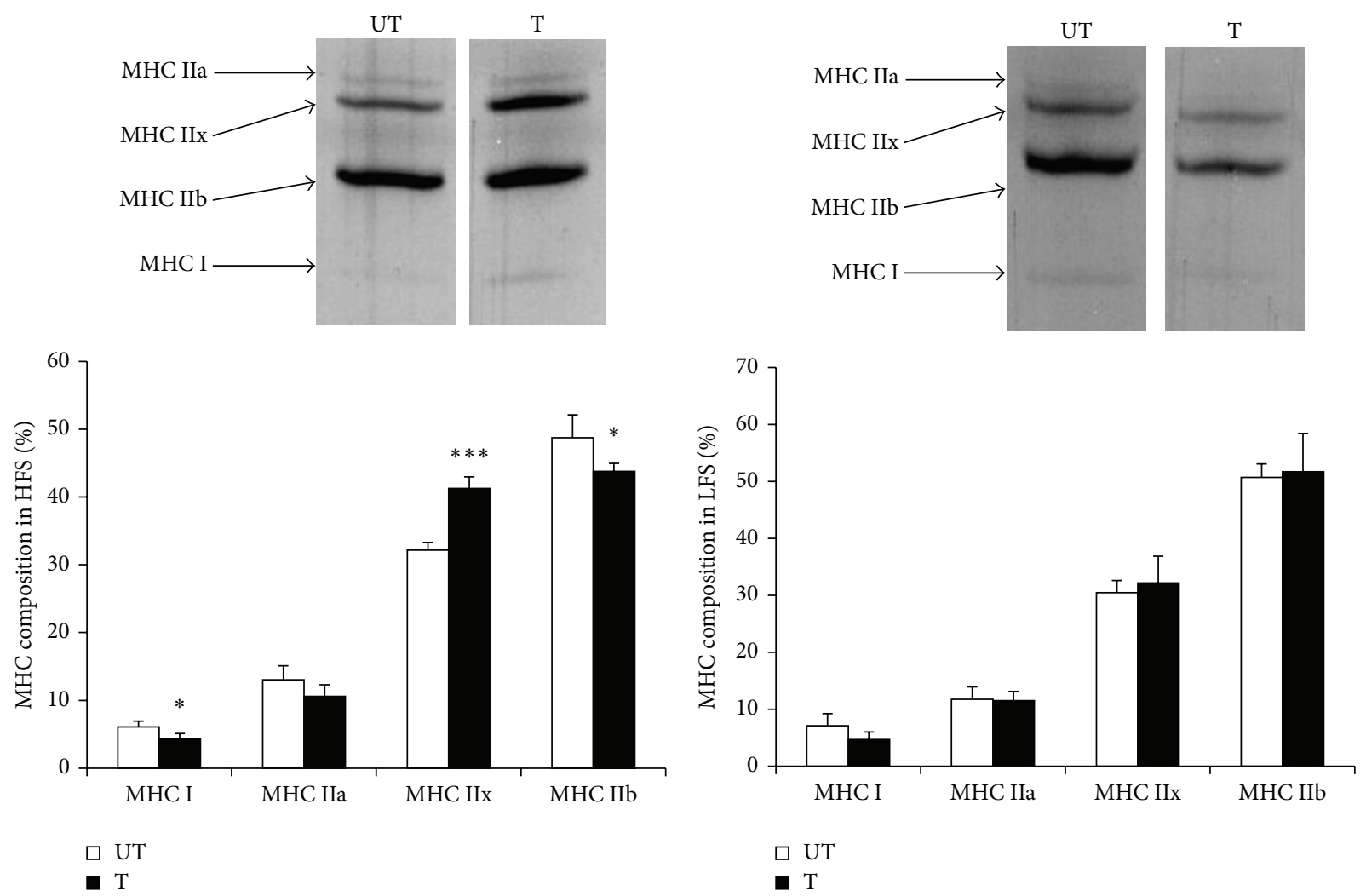

(a)

(b)

FIGURE 5: Myosin heavy chain composition after 6 sessions of training. (a) Myosin heavy chain composition after 6 sessions of training in HFS. (b) Myosin heavy chain composition after 6 sessions of training in LFS. HFS: high-frequency electrical stimulation group. LFS: lowfrequency electrical stimulation group. T: the trained leg. UT: the untrained leg. MHC: myosin heavy chain. All values are the mean \pm SD. ${ }^{*} P<0.05$ and ${ }^{* * *} P<0.001$, versus UT.

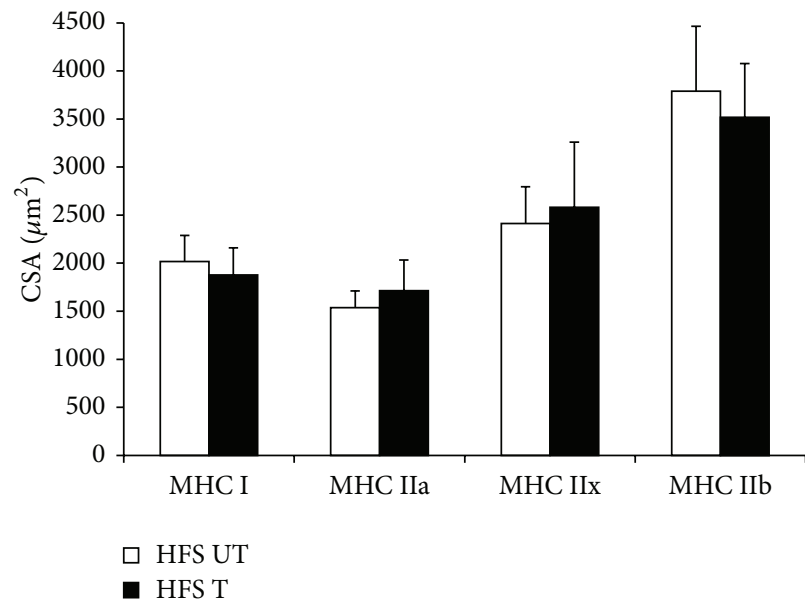

(a)

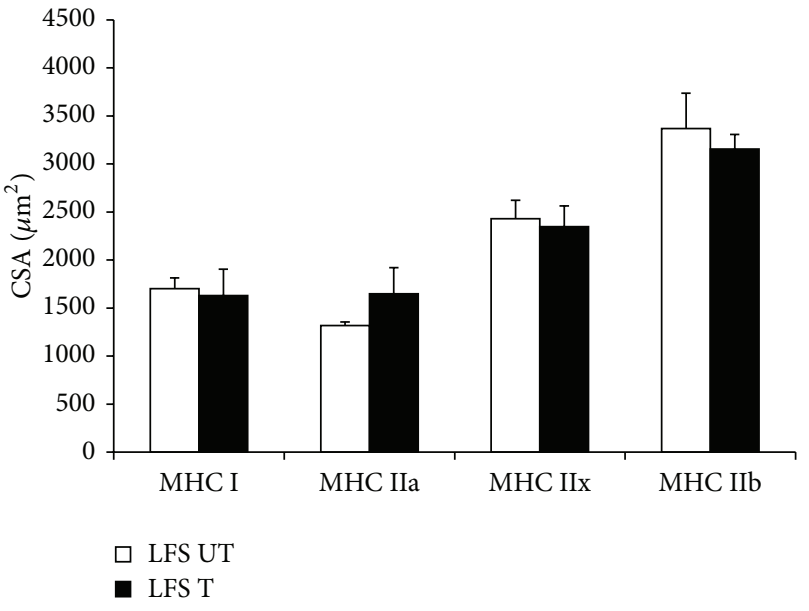

(b)

Figure 6: CSA measurement. (a) The CSA after 6 sessions of training in HFS. (b) The CSA after 6 sessions of training in LFS. HFS: highfrequency electrical stimulation group. LFS: low-frequency electrical stimulation group. T: the trained leg. UT: the untrained leg. CSA: crosssectional area. All values are the mean \pm SD. 
TABLE 1: Physiological characteristics after six sessions of training employing low- and high-electrical frequencies.

\begin{tabular}{lcc}
\hline & HFS & LFS \\
\hline $\begin{array}{l}\text { Body weight (g) } \\
\text { Medial gastrocnemius } \\
\text { wet weight (mg) }\end{array}$ & $366.36 \pm 22.17^{* *}$ & $362.31 \pm 12.36^{* * *}$ \\
$\quad \mathrm{~T}$ & $887.10 \pm 20.77$ & $883.92 \pm 37.50$ \\
$\quad \mathrm{UT}$ & $832.02 \pm 35.54^{* *}$ & $823.08 \pm 37.48^{* * *}$ \\
Medial gastrocnemius & & \\
wet weight/Body & & \\
weight & & \\
$\quad$ T & & $2.44 \pm 0.09$ \\
$\quad$ UT & $2.43 \pm 0.13$ & $2.27 \pm 0.05$ \\
\% increase of muscle & & \\
wet weight \\
$\begin{array}{l}\text { (T versus UT) } \\
\text { \% increase of } \\
\text { myofibrillar protein } \\
\text { content } \\
\text { (T versus UT) }\end{array}$
\end{tabular}

HFS: high-frequency electrical stimulation, LFS: low-frequency electrical stimulation, T: trained leg, and UT: untrained leg. Values are mean \pm SD. ${ }^{*} P<0.05,{ }^{* *} P<0.01,{ }^{* * *} P<0.001$ versus UT.

\section{Discussion}

In this study, we found that 1 bout of intermittent lowfrequency electrical stimulations successfully activated one of the anabolic responses of ERK signaling. We also found that chronic bouts of LFS significantly increased the muscle mass and myofibrillar protein. This increase was comparable to that obtained with HFS, which has already been shown as training for muscle hypertrophy. We also found that MHCs and activated signaling molecules differed in composition in LFS- and HFS-induced hypertrophies. In the following discussion, we will discuss the potential biological mechanisms underlying LFS-induced muscle hypertrophy.

A number of studies have demonstrated that the level of p70S6K phosphorylation can be used as an indicator of muscle hypertrophy and/or anabolic response. Our examination found that a significant increase in phosphorylated p70S6K was only observed in the HFS group. Mitchell et al. [6] reported that the level of p70S6K phosphorylation does not increase during low-intensity combined with highvolume training, yet our data showed skeletal muscle hypertrophy occurring with LFS without the phosphorylation of p70S6K. This may be explained by the possibility that our experimental model of LFS represents this low-intensity and high-volume training condition. We also investigated the phosphorylation level of ERK 1/2, a member of the MAPK signaling pathway and found it to be increased only in the LFS group, immediately after training. Since the phosphorylation of p70S6K is elicited mainly in type II fibers $[9,26]$, the activation of type II fibers and the increase in MHC IIx might be related to signal transduction during HFS. As ERK $1 / 2$ expression did not demonstrate fiber type specificity, our observation that the MHC composition was not affected by
LFS stimulation suggests that a relatively high activation of ERK 1/2 might lead to unspecific hypertrophy in rat medial gastrocnemius muscles. Taken together, we speculate that mTOR signaling is a major contributor to HFS-induced hypertrophy, and that MAPK signaling plays a role in LFSinduced hypertrophy.

A key finding in the current study was that the 6session trainings with LFS successfully induced a significant increase in muscle mass and myofibrillar protein contents, and that this increase was determined to be the same in the muscle stimulated with HFS. Since 1 bout of LFS exercise induces ERK phosphorylation, chronic bout of LFS is an accumulation of such acute anabolic response. However, we failed to observe a significant increase in the CSA analysis after chronic bouts of training. This may be ascribed to the fact that 6-session training is a comparatively shorter time than previous studies $[17,27]$.

In Section 1, we raised the possibility that NMES randomly activates type I and type II fibers, leading to muscle hypertrophy. In this study, we performed PAS staining to investigate which fibers were activated by NMES. The results showed that muscle fibers were randomly activated by both LFS and HFS, independent of the exerted force. The manner of muscle fiber activation under electrical stimulation was similar to that of a previous report, suggesting that NMES activates muscle fibers at random [15]. Taken together, we consider that both type I and type II fibers are activated under both HFS and LFS treatments.

In both the LFS and HFS groups, the intramuscular glycogen was mainly depleted in the fibers located in the white portion in the medial gastrocnemius muscle. In the red portion, intramuscular glycogen was depleted in the HFS group, but clear staining was observed after LFS treatment. The HFS protocol in our experiment is essentially the same as that used by previous studies $[18,27,28]$, and this condition led to a supramaximal activation of gastrocnemius muscles. On the other hand, we observed that the exerted force and work volume in the LFS group was about less than $50 \%$ of that in the HFS group. Such differences in exerted force could be related to differences in glycogen consumption.

As seen in the immunostaining of type II fibers, the gastrocnemius muscle consisted mainly of type II fibers, especially in the white portion. This is the same result as shown by previous studies [17, 24, 27]. Since the major fibers were of type II in the white portion, random activation dominantly recruited type II fibers, regardless of the electrical frequency. Since type II fiber activation is a key event in muscle hypertrophy, LFS might successfully induce muscle hypertrophy in gastrocnemius muscles.

Recently, muscle hypertrophy was reported to be induced by moderate- and/or low-intensity (20-50\% of $1 \mathrm{RM}$ ) resistance training, with blood-flow restriction [29-32]. Moreover, Fujita et al. [33] have also reported that an increase of the muscle protein synthesis rate occurs in the $3 \mathrm{~h}$ following low-intensity, blood-flow-restricted resistance exercise. More recent studies have determined that low intensity training to the point of fatigue without blood-flow restriction also elicits a higher protein synthesis rate and muscle hypertrophy $[5,6]$. This supports the idea that a recruitment of 
type II fibers occurs under fatigable conditions, even if the weight lifted is low. It is obvious that human studies are far from the animal models, and our LFS model underscores the theory that activation of type II fibers is a key factor in achieving muscle hypertrophy without high exerted force.

Various exercise methods can induce fiber type alteration. We examined whether training could stimulate fiber type changes, by modifying the myosin heavy chain composition. As previously reported, during HFS, MHC IIx expression was increased in the medial gastrocnemius muscle, whereas MHC IIb and MHC I expressions were decreased [17, 27]. Unexpectedly, we found that LFS did not change the MHC composition. Normally, ATP-consuming muscle contraction induces the switch from a fast- to a slow-twitch fiber activation. Muscle hypertrophy, with no change in the MHC composition, suggests that all muscle fibers are equally enlarged, independent of fiber type. We also speculate that the groups of activated signaling molecules might differ between LFS and HFS. In the acute experiment, we found that $\mathrm{p} 70 \mathrm{~S} 6 \mathrm{~K}$ was activated by HFS, and ERK $1 / 2$ was activated by LFS. Since the phosphorylation of p70S6K elicited mainly type II fibers $[9,26]$, the activation of type II fibers and the increase in MHC IIx might be related to the signal transduction induced by HFS. As ERK $1 / 2$ expression did not demonstrate fiber-type specificity, our observation that the MHC composition was not affected by LFS suggests that a relatively high activation of ERK 1/2 might lead to unspecific hypertrophy in the rat medial gastrocnemius muscle. It is also important to evaluate whether functional differences exist between muscles treated with different electrical frequencies.

For humans, skeletal muscle activation by NMES is a method of physical therapy aimed at augmenting and/or maintaining skeletal muscle performance. In clinical settings, high-frequency NMES is mostly dedicated to the improvement of the muscular strength and is similar to resistance training [34]. Conversely, low-frequency NMES improves the metabolic and histochemical characteristics of the skeletal muscle and is thought to mimic endurance training [35]. Our data suggest that electrical stimulation of the skeletal muscle with low-force generation can be beneficial in achieving muscle hypertrophy without the pain associated with high frequency electrical stimulation.

\section{Conclusions}

In summary, our present study demonstrates that muscle activation by electrical stimulation recruits type II fibers independently of frequency and that electrical stimulation without high force generation results in muscle hypertrophy. This finding may be applicable to both athletic conditioning as well as to clinical care for sports injuries and muscle atrophy.

\section{Conflict of Interests}

All authors declare that they have no conflict of interests.

\section{Acknowledgment}

This study was supported by the Grant-in-Aid for Scientific Research (C; 00307993) from KAKENHI.

\section{References}

[1] American College of Sports Medicine Position Stand, "Progression models in resistance training for healthy adults," Medicine \& Science in Sports \& Exercise, vol. 41, no. 3, pp. 687-708, 2009.

[2] J. D. MacDougall, G. C. B. Elder, and D. G. Sale, "Effects of strength training and immobilization on human muscle fibers," European Journal of Applied Physiology and Occupational Physiology, vol. 43, no. 1, pp. 25-34, 1980.

[3] P. Aagaard, J. L. Andersen, P. Dyhre-Poulsen et al., "A mechanism for increased contractile strength of human pennate muscle in response to strength training: changes in muscle architecture," The Journal of Physiology, vol. 534, no. 2, pp. 613623, 2001.

[4] G. E. McCall, W. C. Byrnes, A. Dickinson, P. M. Pattany, and S. J. Fleck, "Muscle fiber hypertrophy, hyperplasia, and capillary density in college men after resistance training," Journal of Applied Physiology, vol. 81, no. 5, pp. 2004-2012, 1996.

[5] N. A. Burd, D. W. D. West, A. W. Staples et al., "Low-load high volume resistance exercise stimulates muscle protein synthesis more than high-load low volume resistance exercise in young men," PLoS ONE, vol. 5, no. 8, Article ID e12033, 2010.

[6] C. J. Mitchell, T. A. Churchward-Venne, D. D. West et al., "Resistance exercise load does not determine training-mediated hypertrophic gains in young men," Journal of Applied Physiology, 2012.

[7] S. C. Bodine, T. N. Stitt, M. Gonzalez et al., "Akt/mTOR pathway is a crucial regulator of skeletal muscle hypertrophy and can prevent muscle atrophy in vivo," Nature Cell Biology, vol. 3, no. 11, pp. 1014-1019, 2001.

[8] D. R. Bolster, N. Kubica, S. J. Crozier et al., "Immediate response of mammalian target of rapamycin (mTOR)-mediated signalling following acute resistance exercise in rat skeletal muscle," Journal of Physiology, vol. 553, no. 1, pp. 213-220, 2003.

[9] K. Baar and K. Esser, "Phosphorylation of p70(S6k) correlates with increased skeletal muscle mass following resistance exercise," American Journal of Physiology, vol. 276, no. 1, pp. C120C127, 1999.

[10] Y.-H. Song, M. Godard, Y. Li, S. R. Richmond, N. Rosenthal, and P. Delafontaine, "Insulin-like growth factor I-mediated skeletal muscle hypertrophy is characterized by increased mTORp70S6K signaling without increased Akt phosphorylation," Journal of Investigative Medicine, vol. 53, no. 3, pp. 135-142, 2005.

[11] H. K. R. Karlsson, P.-A. Nilsson, J. Nilsson, A. V. Chibalin, J. R. Zierath, and E. Blomstrand, "Branched-chain amino acids increase p70S6k phosphorylation in human skeletal muscle after resistance exercise," American Journal of Physiology, vol. 287, no. 1, pp. E1-E7, 2004.

[12] C. Wretman, A. Lionikas, U. Widegren, J. Lännergren, H. Westerblad, and J. Henriksson, "Effects of concentric and eccentric contractions on phosphorylation of MAPKerk1/2 and MAPKp38 in isolated rat skeletal muscle," Journal of Physiology, vol. 535, no. 1, pp. 155-164, 2001.

[13] D. Williamson, P. Gallagher, M. Harber, C. Hollon, and S. Trappe, "Mitogen-activated protein kinase (MAPK) pathway activation: effects of age and acute exercise on human skeletal muscle," Journal of Physiology, vol. 547, no. 3, pp. 977-987, 2003. 
[14] M. Yu, N. K. Stepto, A. V. Chibalin et al., "Metabolic and mitogenic signal transduction in human skeletal muscle after intense cycling exercise," Journal of Physiology, vol. 546, no. 2, pp. 327$335,2003$.

[15] C. M. Gregory, W. Dixon, and C. S. Bickel, "Impact of varying pulse frequency and duration on muscle torque production and fatigue," Muscle and Nerve, vol. 35, no. 4, pp. 504-509, 2007.

[16] R. Ogasawara, K. Kobayashi, A. Tsutaki et al., "mTOR signaling response to resistance exercise is altered by chronic resistance training and detraining in skeletal muscle," Journal of Applied Physiology, vol. 114, no. 7, pp. 934-940, 2013.

[17] K. Kobayashi, R. Ogasawara, A. Tsutaki, K. Lee, E. Ochi, and K. Nakazato, "Genetic strain-dependent protein metabolism and muscle hypertrophy under chronic isometric training in rat gastrocnemius muscle," Physiological Research, vol. 61, no. 5, pp. 527-535, 2012.

[18] E. Ochi, K. Nakazato, and N. Ishii, "Effects of eccentric exercise on joint stiffness and muscle connectin (Titin) isoform in the rat hindlimb," Journal of Physiological Sciences, vol. 57, no. 1, pp. $1-6,2007$.

[19] V. J. Caiozzo, E. Ma, S. A. McCue, E. Smith, R. E. Herrick, and K. M. Baldwin, "A new animal model for modulating myosin isoform expression by altered mechanical activity," Journal of Applied Physiology, vol. 73, no. 4, pp. 1432-1440, 1992.

[20] W. H. Kwong and G. Vrbova, "Effects of low-frequency electrical stimulation on fast and slow muscles of the rat," Pflugers Archiv European Journal of Physiology, vol. 391, no. 3, pp. 200-207, 1981.

[21] P. J. Atherton, J. Babraj, K. Smith, J. Singh, M. J. Rennie, and $\mathrm{H}$. Wackerhage, "Selective activation of AMPK-PGC-1 $\alpha$ or PKB-TSC2-mTOR signaling can explain specific adaptive responses to endurance or resistance training-like electrical muscle stimulation," The FASEB Journal, vol. 19, no. 7, pp. 786788, 2005.

[22] C. S. Bickel, C. M. Gregory, and J. C. Dean, "Motor unit recruitment during neuromuscular electrical stimulation: a critical appraisal," European Journal of Applied Physiology, vol. 111, no. 10, pp. 2399-2407, 2011.

[23] L. G. Karagounis, B. B. Yaspelkis III, D. W. Reeder, G. I. Lancaster, J. A. Hawley, and V. G. Coffey, "Contraction-induced changes in TNF $\alpha$ and Akt-mediated signalling are associated with increased myofibrillar protein in rat skeletal muscle," European Journal of Applied Physiology, vol. 109, no. 5, pp. 839848, 2010.

[24] D. Bloemberg and J. Quadrilatero, "Rapid determination of myosin heavy chain expression in rat, mouse, and human skeletal muscle using multicolor immunofluorescence analysis," PLoS ONE, vol. 7, no. 4, Article ID e35273, 2012.

[25] W. Mizunoya, J.-I. Wakamatsu, R. Tatsumi, and Y. Ikeuchi, "Protocol for high-resolution separation of rodent myosin heavy chain isoforms in a mini-gel electrophoresis system," Analytical Biochemistry, vol. 377, no. 1, pp. 111-113, 2008.

[26] R. Koopman, A. H. G. Zorenc, R. J. J. Gransier, D. CameronSmith, and L. J. C. van Loon, "Increase in S6K1 phosphorylation in human skeletal muscle following resistance exercise occurs mainly in type II muscle fibers," American Journal of Physiology, vol. 290, no. 6, pp. E1245-E1252, 2006.

[27] F. Haddad, A. X. Qin, M. Zeng, S. A. McCue, and K. M. Baldwin, "Effects of isometric training on skeletal myosin heavy chain expression," Journal of Applied Physiology, vol. 84, no. 6, pp. 2036-2041, 1998.

[28] E. Ochi, K. Nakazato, and N. Ishii, "Muscular hypertrophy and changes in cytokine production after eccentric training in the rat skeletal muscle," Journal of Strength and Conditioning Research, vol. 25, no. 8, pp. 2283-2292, 2011.

[29] T. Abe, C. F. Kearns, and Y. Sato, "Muscle size and strength are increased following walk training with restricted venous blood flow from the leg muscle, Kaatsu-walk training," Journal of Applied Physiology, vol. 100, no. 5, pp. 1460-1466, 2006.

[30] D. R. Moore, K. A. Burgomaster, L. M. Schofield, M. J. Gibala, D. G. Sale, and S. M. Phillips, "Neuromuscular adaptations in human muscle following low intensity resistance training with vascular occlusion," European Journal of Applied Physiology, vol. 92, no. 4-5, pp. 399-406, 2004.

[31] Y. Takarada, Y. Sato, and N. Ishii, "Effects of resistance exercise combined with vascular occlusion on muscle function in athletes," European Journal of Applied Physiology, vol. 86, no. 4, pp. 308-314, 2002.

[32] M. Shinohara, M. Kouzaki, T. Yoshihisa, and T. Fukunaga, "Efficacy of tourniquet ischemia for strength training with low resistance," European Journal of Applied Physiology and Occupational Physiology, vol. 77, no. 1-2, pp. 189-191, 1998.

[33] S. Fujita, T. Abe, M. J. Drummond et al., "Blood flow restriction during low-intensity resistance exercise increases S6K1 phosphorylation and muscle protein synthesis," Journal of Applied Physiology, vol. 103, no. 3, pp. 903-910, 2007.

[34] T. Hortobágyi and N. A. Maffiuletti, "Neural adaptations to electrical stimulation strength training," European Journal of Applied Physiology, vol. 111, no. 10, pp. 2439-2449, 2011.

[35] R. Theriault, M. R. Boulay, G. Theriault, and J. A. Simoneau, "Electrical stimulation-induced changes in performance and fiber type proportion of human knee extensor muscles," European Journal of Applied Physiology and Occupational Physiology, vol. 74, no. 4, pp. 311-317, 1996. 

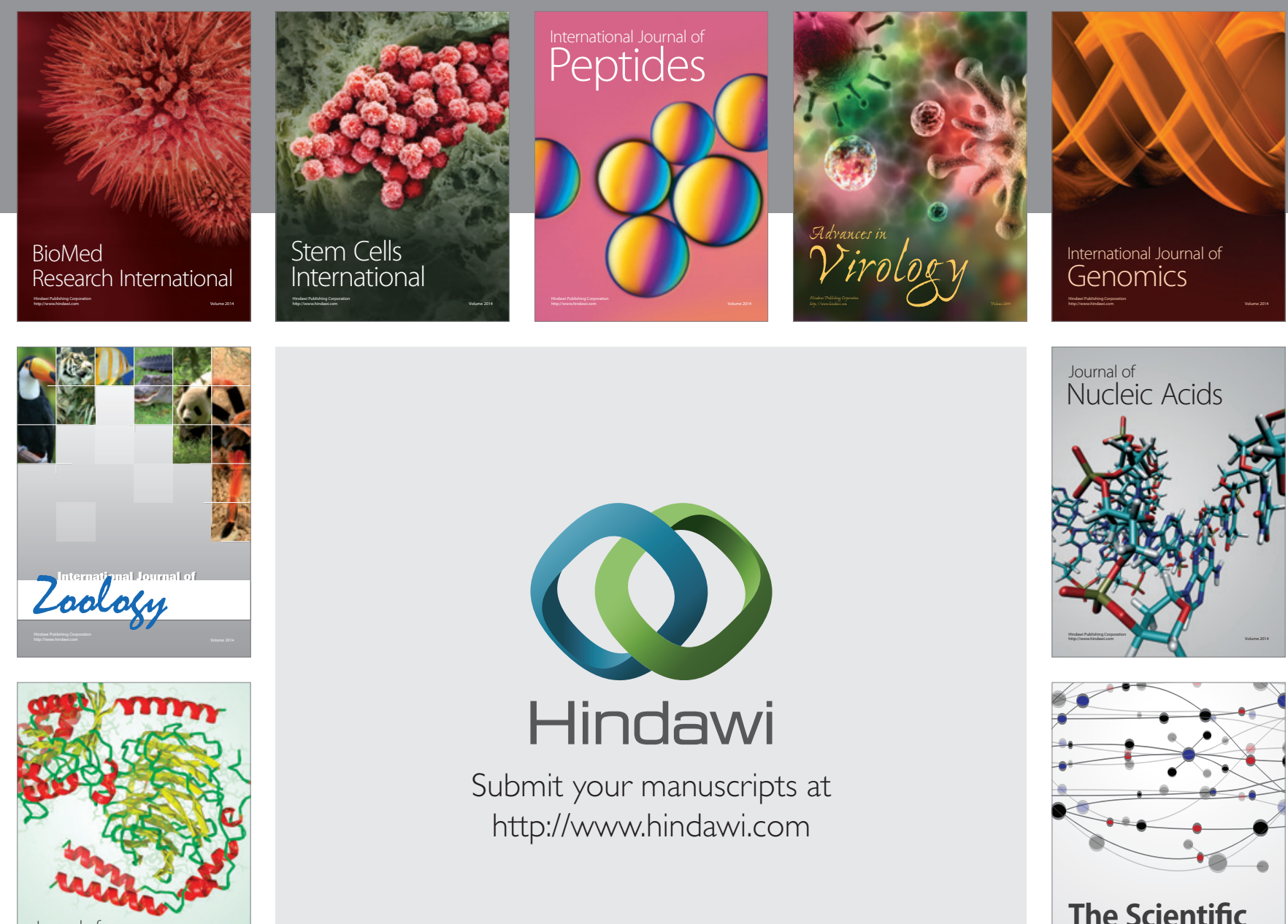

Submit your manuscripts at

http://www.hindawi.com

Journal of
Signal Transduction
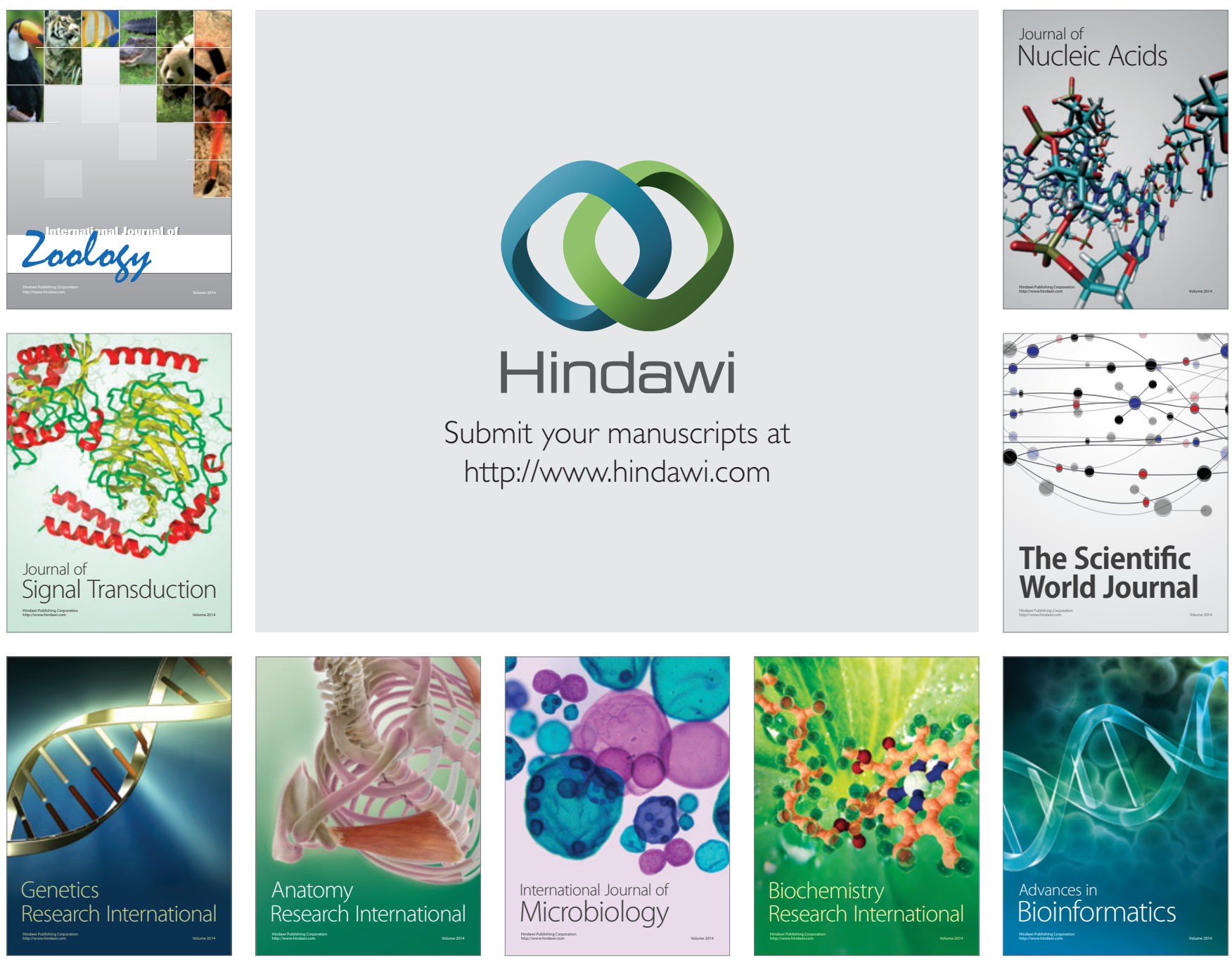

The Scientific World Journal
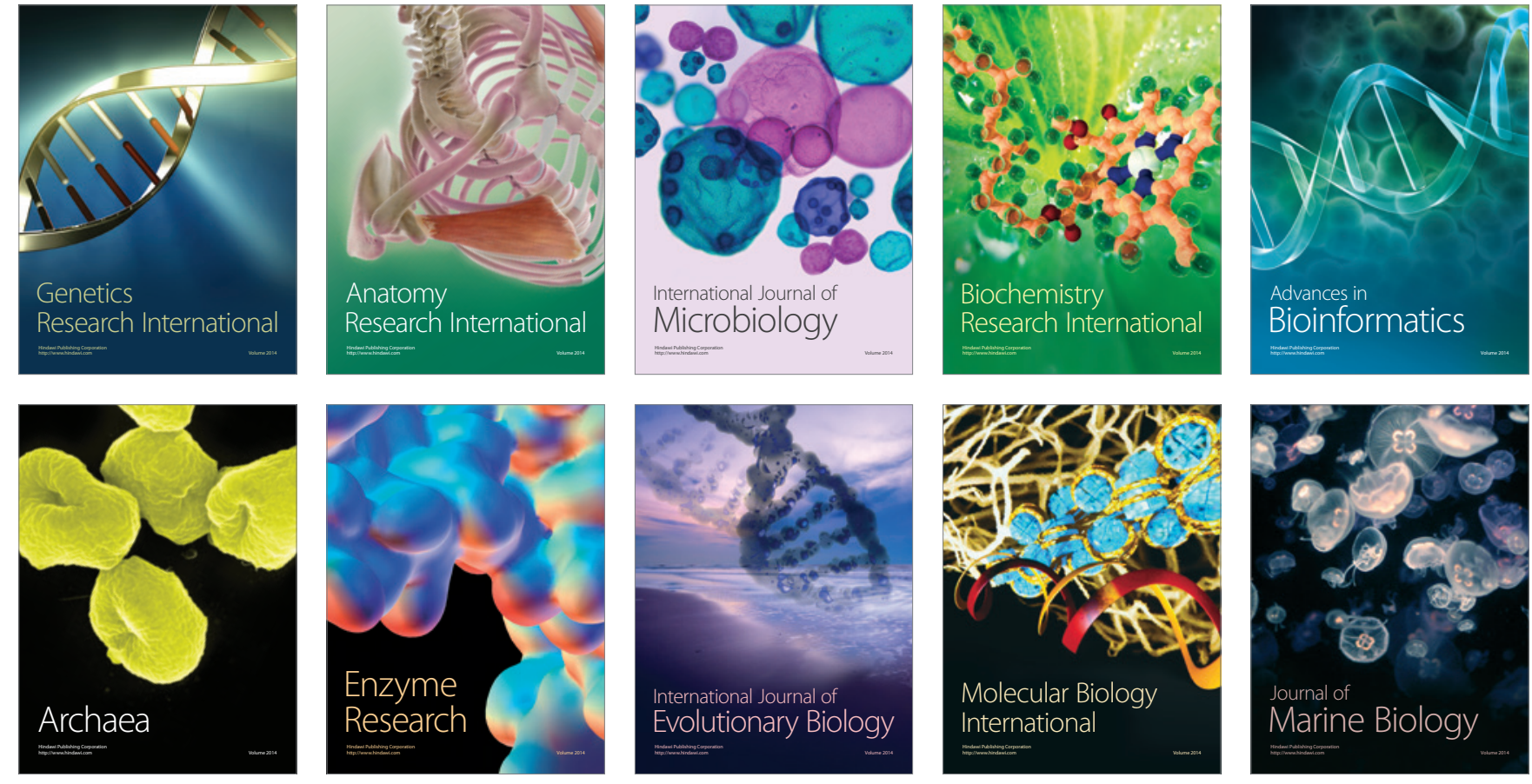